\title{
Atomistic Shock Hugoniot simulation of single-crystal copper
}

E. M. Bringa, J. U. Cazamias, P. Erhart, J. Stolken, N. Tanushev, B. D. Wirth, R. E. Rudd, M. J. Caturla

September 10, 2004

Applied Physics Letters 
This document was prepared as an account of work sponsored by an agency of the United States Government. Neither the United States Government nor the University of California nor any of their employees, makes any warranty, express or implied, or assumes any legal liability or responsibility for the accuracy, completeness, or usefulness of any information, apparatus, product, or process disclosed, or represents that its use would not infringe privately owned rights. Reference herein to any specific commercial product, process, or service by trade name, trademark, manufacturer, or otherwise, does not necessarily constitute or imply its endorsement, recommendation, or favoring by the United States Government or the University of California. The views and opinions of authors expressed herein do not necessarily state or reflect those of the United States Government or the University of California, and shall not be used for advertising or product endorsement purposes. 


\title{
Atomistic shock Hugoniot simulation of single-crystal copper
}

\author{
E.M. Bringa
}

Chemistry and Materials Sciences, Lawrence Livermore National Laboratory, Livermore CA

\author{
J.U. Cazamias \\ Nonproliferation, Arms Control, and International Security, \\ Lawrence Livermore National Laboratory, Livermore CA \\ P. Erhart \\ Institut für Materialwissenschaft, Technische Universität Darmstadt, Darmstadt, Germany \\ J. Stölken \\ Engineering, Lawrence Livermore National Laboratory, Livermore \\ N. Tanushev \\ Mathematics Department, University of California, Los Angeles \\ B.D. Wirth \\ Nuclear Engineering Department, University of California, Berkeley \\ R.E. Rudd \\ Physics and Advanced Technologies, \\ Lawrence Livermore National Laboratory, Livermore
}

\author{
M.J. Caturla \\ Departament de Fisica Aplicada, Universitat d'Alacant, E-03690 Alacant, Spain
}




\begin{abstract}
Planar shock waves in single-crystal copper were simulated using non-equilibrium molecular dynamics with a realistic embedded atom potential. The simulation results are in good agreement with new experimental data presented here, for the Hugoniot of single-crystal copper along $\langle 100\rangle$. Simulations were performed for Hugoniot pressures in the range $2 \mathrm{GPa}-800 \mathrm{GPa}$, up to well above the shock induced melting transition. Large anisotropies are found for shock propagation along $\langle 100\rangle,\langle 110\rangle$, and $\langle 111\rangle$, with quantitative differences from pair potentials results. Plastic deformation starts at $U_{p} \gtrsim 0.75 \mathrm{~km} / \mathrm{s}$, and melting occurs between 200 and $220 \mathrm{GPa}$, in agreement with the experimental melting pressure of polycrystalline copper. The Voigt and Reuss averages of our simulated Hugoniot do not compare well below melting with the experimental Hugoniot of polycrystalline copper. This is possibly due to experimental targets with preferential texturing and/or a much lower Hugoniot elastic limit.
\end{abstract}

PACS numbers: 02.70.Ns, 05.70.Ln, 52.35.Tc, 62.50.+p, 64.30.+t

Keywords: copper single-crystal, shock waves, molecular dynamics, high strain-rate phenomena 


\section{INTRODUCTION}

Shock waves have long been used to study the equation of state of materials at extreme conditions [1-3]. In addition, experiments on shock-induced plasticity and fracture have provided useful insight into material deformation and failure [4]. However, a number of issues are still not well understood. The constitutive equations are typically based on an equation of state that assumes isotropic material response, an assumption which is certainly not true for single crystals, and may even pose a problem for textured polycrystals. Furthermore, in the context of plasticity a strong indication of limitations of our understanding is given by the classic Frost and Ashby deformation maps which show an "unexplored region" for deformation occurring at strain rates higher than $10^{6} / \mathrm{s}$ [5]. Laser-induced shocks [6] provide a new way to produce very high strain rates, up to $\sim 10^{10} / \mathrm{s}$ for experiments to be carried out in the near future at the National Ignition Facility (NIF). In addition, recent data for single crystals $[7,8]$ suggest that the plastic response in Cu occurs rapidly, but even with the fast dislocation motion in $\mathrm{Cu}$, the kinetics are likely to be important on timescales that will soon be accessible in experiment ( $\sim 100 \mathrm{ps})$.

There are current limitations on our abilities to model dynamic shock processes accurately, but advances in computing have greatly extended the capabilities of numerical simulations. In particular, molecular-dynamics (MD) simulations solve Newton's equations of motion for a collection of interacting particles over a number of time steps [9]. The size of the simulated system is limited by the number of available processors, and simulations of $10^{7}-10^{10}$ atoms are now possible using the largest parallel computers. MD simulations generally probe strain rates well above $10^{6} / \mathrm{s}$ and, therefore, are a natural complement to understand atomic level mechanisms during shock compression. Linking to longer time and larger length scales could be accomplished within a multiscale framework, for instance by dislocation dynamics informed by MD simulations and coupled to a finite elements mesh $[10]$.

Most atomistic shock simulation studies have investigated single crystal response to shocks along the $\langle 100\rangle$ direction [11-13]. On the other hand, nearly all experimental studies of shock waves in metals have been performed with polycrystalline samples [1-3]. Clearly, in the majority of metals, the directional anisotropies in single crystals will give rise to direction-dependent Hugoniot relationships. Such anisotropies could be mapped by MD 
simulations and provide a guide to future and ongoing [7, 8] experimental efforts. Germann et al. [14] presented results for the Hugoniot of a Lennard Jones (LJ) fcc crystal showing a rich variety of behaviors depending on shock orientation.

A shock wave can be produced if a surface force, to which we will refer as a piston, is steadily applied to a material on one side. In the limit of zero piston pressure we expect to obtain a shock velocity equal to the longitudinal sound speed. The longitudinal/transverse sound speeds, in an isotropic elastic medium, can be calculated as $c_{o L / T}=\sqrt{A_{L / T} K / \rho}$, where $K$ is the bulk modulus, $\rho$ is the density of the material, and $A_{L}=\frac{3(1-\nu)}{(1+\nu)} ; A_{T}=\frac{3(1-2 \nu)}{2(1+\nu)}$, with $\nu$ the Poisson ratio. For non-zero piston pressure, the Hugoniot relationships [15], i.e. conservation of mass, momentum and energy at the shock front, apply and give the shock speed $U_{s}$ as $U_{s}=U_{p} / \epsilon$, where $U_{p}$ is the particle velocity and $\epsilon=\left(1-\rho_{0} / \rho\right)$ is the volumetric compressive strain. In the strong shock regime, when the plastic wave has overdriven the elastic wave, it is typically found that

$$
U_{s}=U_{o}+s_{1} U_{p}
$$

where $s_{1}$ is a constant in the range $0.5-2.5$, and $U_{o} \sim c_{o}$, the bulk sound speed. Of course, if there is a phase transition, the slope of the Hugoniot may change, and this has often been used as a diagnostic to detect such a transition. Using reasonable approximations for a model solid, it can be shown that the Grüneisen parameter $\gamma_{G}$ is a function only of the compression, $\epsilon$, and $s_{1}[16]$. For the limit of $\epsilon=0, \gamma_{G}=2 s_{1}-1[15,16]$. On the other hand, the fact that $s_{1}$ is a constant for strong shocks implies that there is a limiting compression value, $\epsilon=1-1 / s_{1}$. The Grüneisen parameter at this compression limit is often given as $\gamma_{G}=2\left(s_{1}-1\right)$, which is smaller than the previous value by 1 [16].

The relatively simple picture above is no longer true for an anisotropic solid. For instance, for propagation along $\langle 100\rangle$ in a cubic crystal, $c_{o L\langle 100\rangle}=\sqrt{c_{11} / \rho}$ and $c_{o T\langle 100\rangle}=\sqrt{c_{44} / \rho}$, where $c_{i j}$ are the elastic constants of the cubic crystal. Experimentally, for $\mathrm{Cu}$ the asymmetry is large, $c_{o L\langle 100\rangle} / c_{o T\langle 100\rangle}=1.49$, with the anisotropy ratio $A=2 c_{44} /\left(c_{11}-c_{12}\right)=3.21$, compared to $A=1$ for the isotropic case [17]. The 'isotropic' Grüneisen parameter is no longer applicable. One needs to calculate an anisotropic Grüneisen parameter and also needs a direction-dependent equation of state [18].

Typically, three regions may be identified in the $U_{s}-U_{p}$ Hugoniot [14]. For $U_{p}<U_{p H E L}$, only an elastic front is observed. At the Hugoniot Elastic Limit (HEL), a plastic wave 
appears, and this wave may be under-driven, moving slower than the elastic wave up to $U_{p}<U_{p S}$. For $U_{p} \geq U_{p S}$ the plastic wave overdrives the elastic front, which is not stable. In this last regime the velocity of the elastic front is not the same as the one of the plastic front, but lower. The region $U_{p H E L} \leq U_{p}<U_{p S}$ may be narrow or not exist at all, depending on the material. For any crystal direction, in the limit $U_{p} \rightarrow 0, U_{s} \rightarrow c_{o L}>c_{o}$. On the other hand, in the strong shock regime Eq. 1 is valid. Since $c_{o L}>c_{o}$, there must be a change in slope for velocities below the strong shock regime.

The dependence of the plastic wave speed on orientation may be understood in terms of both elastic and plastic anisotropy. Using the result of Drugan [19] the steady-state shock behavior may be interpreted using the solution for a smooth wave. One such smooth wave solution for a rate-independent elasto-plastic material, given by Lubliner [20], gives the wavespeed as:

$$
U_{s}=\sqrt{A_{1} / \rho},
$$

with

$$
A_{1}=K+(2 / 3) h-2 h^{2} /(3 h+6 \mu)
$$

where $\mu$ is the shear modulus, and $h$ is the plastic modulus (hardening rate). For $h \ll \mu$ (the usual case), this reduces to:

$$
A_{1} \sim K+(2 / 3) h
$$

Thus the plastic wave speed depends in general upon bulk modulus, the shear modulus, and the plastic modulus. Although the bulk modulus of a material with cubic symmetry is independent of loading direction, both the shear and plastic response depend quite strongly on oriention. For copper, the shear modulus varies by over a factor of three depending on orientation [21] while the strain hardening depends strongly on loading direction [22]. As the shock strength increases the hardening response saturates resulting in less plastic anisotropy. Given these combined effects of elastic and plastic anisotropy, it is expected that the shock response of single crystals (and textured polycrystals) should be anisotropic.

In principle, if one is only interested in the Hugoniot curve for polycrystals, one can obtain very good agreement with experiments by calculating an accurate bulk modulus as function of the pressure and temperature [23], and any potential giving that functional form 
will also suffice. This is because the constant $c_{0}$ is equal to the bulk sound velocity at zero pressure $(\sqrt{K / \rho})$, and the Grüneisen parameter, which gives $s_{1}$, can be related to the pressure derivative of the bulk modulus [18]. This approach could be extended to single crystals calculating the appropriate elastic constants as a function of pressure. With this information one can obtain the equilibrium states that form the Hugoniot. However, this approach, or the use of equilibrium MD calculations [24], does not provide any information on when will plastic behavior start, what kind of plastic behavior will be found, etc. This is where non-equilibrium MD simulations play an important role, with the disadvantage that they are computer intensive. Constrained techniques, like the "Hugoniostat" [13], can bridge these two approaches.

In this work, we present a comparison of experiments and simulation for the $\langle 100\rangle$ shock Hugoniot of solid $\mathrm{Cu}$, and simulation results for the Hugoniot along other crystalline directions, finally averaging these results to compare with experiments on polycrystals. The overall behavior for an fcc LJ solid presented by Germann et al. [14] is qualitatively similar to the one seen here for EAM potentials, but important quantitative differences arise as discussed below.

\section{A. Experiments}

Plate impact experiments (1D strain) were performed on single crystal copper using LLNL's $35 \mathrm{~mm}$ light gas gun. The flyer plate (impactor) of polycrystalline copper was $1.5 \mathrm{~mm}$ thick. The target plate was $5 \mathrm{~mm}$ thick. The experiments were designed to look at the spall behavior of copper and measured free surface velocity using laser interferometry (VISAR) [25]. Hugoniot measurements were backed out of the data using an impedance matching technique. At the flyer-target interface, pressure and particle velocity are identical in both samples. Using the Hugoniot relation $P=\rho_{0} U_{s} U_{p}$, where $P$ is the Hugoniot pressure and $\rho_{0}$ is the initial density, allows one to calculate the shock velocity of the target assuming that one knows the Hugoniot of the polycrystalline copper flier [a standard $-U_{s}=3.94 \mathrm{~km} / \mathrm{s}+$ $\left.1.489 U_{p}(\mathrm{~km} / \mathrm{s})\right]$ and the particle velocity of the target which is taken to be one half the free surface velocity. 


\section{B. MD Simulations}

The simulations were performed with the MDCASK code [26], adapted to simulate shock waves [27]. A box elongated along the $\mathrm{z}$ direction was equilibrated during several ps at $1.5 \mathrm{~K}$, using periodic boundaries only along the $\mathrm{x}$ and $\mathrm{y}$ directions. A square pulse shock wave was applied along $\widehat{z}$ by adding an external force to few planes of atoms on one of the free surfaces. The applied force was kept constant throughout these simulations, although a time dependent profile could be applied. Velocity profiles were analyzed at subsequent times to calculate both $U_{p}$ and $U_{s}$. Following a transient stage, typically around $0.5 \mathrm{ps,} \mathrm{the}$ elastic shock profiles reached a steady state, allowing a determination of $U_{p}$ and $U_{s}$ with errors generally smaller than $5 \%$. Both plastic and elastic fronts were seen in the simulations above a "plastic threshold", as for the LJ simulations [14]. Our simulations, employing a planar, but fully 3-D geometry, are quite different from "equilibrium" simulations where the Hugoniot is extracted from a "hydrostatic" compression at relatively small system sizes [24]. Thus, we can easily capture the evolution of the shock without the assumption of local thermal equilibrium, together with the detailed dynamics of the deformation originating at the front.

Most "non-equilibrium" shock simulation studies of fec solids have used pair potentials, including LJ [11-14], Morse [28], exponential-6 [29], etc. Taylor and Dodson recently presented results on the Hugoniot of EAM Cu along $\langle 100\rangle$ using an embedded-atom (EAM) potential, for $U_{p} \in(0,2.5)$ and a target with $4 \times 4 \times 30$ unit cells [28]. Ryazanov et al. [30] have also presented some simulations of point defect formation by multiple weak shock waves in EAM Cu, and Kum [31] has shown results for shock waves at a single $U_{p}$ above the plastic limit for EAM and Morse Ni along $\langle 100\rangle,\langle 110\rangle$ and $\langle 111\rangle$. None of these simulations have been compared with experimental data on single crystals.

Here we consider two many body potentials, of the embedded atom form, EAM1 [32] and EAM2 [33]. For shock waves along $\langle 100\rangle$ most simulations were performed with sample sizes of $50 \times 50 \times 200 \mathrm{fcc}$ cells, i.e. $2 \times 10^{6}$ atoms. Using the lattice parameter for $\mathrm{Cu}$,

$a_{o}=3.615 \AA$, the size of the sample was $18.1 \times 18.1 \times 72.3 \mathrm{~nm}^{3}$. Several of the results for EAM2 were obtained for smaller samples with a cross section of $25 \times 25$ cells, and results for these two sample sizes were indistinguishable for the EAM2 potential. Near the threshold for plasticity, plastic shock waves appeared few ps after the shock was applied and it took them 
few additional ps to reach a steady profile, stressing the need to simulate very large samples (more than 100 fcc cells long) for reliable calculation of $U_{s, p l a s t i c}$ with this scheme. For the other two crystalline directions similar sample sizes were used. The following velocities will be given in $\mathrm{km} / \mathrm{s}$, unless noted otherwise.

For any pair potential, $c_{12}=c_{44}$, which fixes the anisotropy. This relationship does not hold experimentally for metals and many body potentials, like EAM, are needed to solve this discrepancy. In addition, it is important to point out that any potential with range $r_{c u t}$ less than the $3^{\text {rd }}$ nearest neighbor distance will have a stable stacking fault (SF) energy equal to zero, $\gamma_{S F}=0$. These potentials may have a non-zero, small, unstable SF energy which will provide a barrier for dislocation nucleation. However, once partial dislocations are nucleated, only $\gamma_{S F}>0$ results in a finite separation between partial dislocations in the fcc crystal, $d_{S F}$. Therefore the behavior of dislocations cannot be accurately simulated for short range potentials. Table I shows some relevant properties for the potentials discussed in the text. $d_{S F}$ is given at zero pressure and may increase under pressure. At a minimum, the lateral size of an MD target must accommodate $d_{S F}$. Using the zero-pressure value for $d_{S F}$ at least 6 unit cells are required in the lateral direction for the EAM2 potential and 22 for the EAM1 potential. EAM2 was fitted to the ab-initio cold curve of Cu, making it particularly attractive for shock simulations.

\section{RESULTS}

In Fig. 1 we have plotted the simulated $\langle 100\rangle$ Hugoniot along with recent single crystal Cu gas-gun data. The EAM2 potential is in good agreement with the experimental results, while the EAM1 potential is shifted slightly to higher values of $U_{s}$. Results for both EAM1 and EAM2 are quite close, indicating that the long range part of the potential (beyond $3^{\text {rd }} \mathrm{NN}$ ) does not play a key role in the $\langle 100\rangle$ Hugoniot below melting. The different values of $\gamma_{S F}$ do not make a significant difference for the elastic Hugoniot, but do impact the plastic deformation at a given $U_{p}$, giving different stacking fault densities for the two potentials at the same piston velocity. The fit to the strong shock regime for a LJ crystal is also included, and shows a large deviation with respect to the experimental data and the EAM results. The LJ potential gives $U_{s}=c_{o L\langle 100\rangle}+1.92 U_{p}[14]$, where the value of $c_{o L\langle 100\rangle}$ could be fit to the experimental data by chosing the appropriate LJ parameters. The EAM2 potential 
gives $U_{s}=(4.1 \pm 0.1)+(1.3 \pm 0.1) U_{p}$ for the range shown in the figure and it is extremely close to the experimental data. The Morse potential provides a good way to compare results for many body and pair potentials, since a LJ potential is very close to a "stiff" Morse with a stiffness parameter $\alpha \sim 6[36,37]$, and the cold curves for the Morse and EAM potentials are comparable to each other when using $\alpha \sim 3$ [38]. One calculation for a Morse potential [36] is also shown in Fig. 1. This value is in good agreement to the Hugoniot results for a Morse potential from ref. [28] using a much smaller system. Since the Morse potential has a much wider well and is softer at short distances, the Hugoniot is expected to be less steep than that of the LJ potential, as shown in Fig. 1. It has also been shown that Exp-6 potential, which is less steep than the LJ potential, gives a less steep Hugoniot than the LJ potential [29].

As expected, when $U_{p} \rightarrow 0, U_{s} \rightarrow c_{o L}=4.3$. Indeed, Fig. 1 shows a small plateau, where the shock velocity stays constant within our error bars as $U_{p} \rightarrow 0$. Plastic response appears at $\left(U_{p} / c_{o L}\right) \sim 0.14$, that corresponds to a compression of about $14 \%$. This is lower than the value for short-range LJ, which was $\left(U_{p} / c_{o L}\right) \sim 0.2$ [14], but happens at about the same compression. The pressure for the HEL is therefore $32 \pm 2 \mathrm{GPa}$, much higher than experimental values [39]. This is not unexpected, since even "perfect" crystals used in the experiments have a pre-existing density of defects [8] that act as nucleation sites for plasticity to begin at a lower stress value. Holian and Lomdahl [11] showed that a non-planar piston leads to a reduced HEL, and preliminary simulations we have carried out including extended defects in our perfect crystal do lower the HEL significantly [40].

The shear stress behind the shock front is only a small fraction of the applied stress, while the hydrostatic pressure is comparable to the applied stress. Therefore, the net shear stress near the plastic threshold is only few GPa, similar to the ideal yield stress of single crystals. It is difficult to calculate the HEL for uniaxial compression, but estimates assuming a perfect elasto-plastic solid without strain rate effects are often used [16, 41, 42]:

$$
\begin{aligned}
\text { HEL } & \approx P_{H}+\frac{2}{3} Y_{0} \\
& \approx \frac{(1-\nu)}{(1-2 \nu)} Y_{0} \\
& \approx\left(\frac{K}{2 \mu}+\frac{2}{3}\right) Y_{0},
\end{aligned}
$$

where $Y_{0}$ is the yield strength of the material, and all quantities are evaluated at the ap- 
propriate hydrostatic pressure, $P_{H}$, which is not known a priori. Using zero pressure values Eq. 5c gives $\sim 4.6 \mathrm{GPa}$, and employing values of $K$ and $\mu$ at $\sim 35 \mathrm{GPa}$ does not give a significant increase in this estimate. The large difference between the HEL from MD and this estimate indicates the limitations of using simplified models for shock behavior in solids.

Figure 2 shows snapshots of the velocity profile along the $z$ direction 3 ps after the shock was applied, for a piston pressure close to $100 \mathrm{GPa}$. In all cases there is an elastic and plastic wave. For the $\langle 100\rangle$ direction both the plastic and elastic wave move at roughly the same speed, and can only be separated examining the structure of the sample behind the shock. For the $\langle 111\rangle$ direction, an elastic precursor is seen, followed by the plastic wave.

In the LJ simulations [14] a number of elastic precursors was seen at zero temperature for shock waves along $\langle 100\rangle$ and for shock waves along $\langle 110\rangle$ when the initial temperature of the sample was below $T_{\text {melt }} / 10$. We observe elastic precursors at finite temperature below the plastic limit for $\langle 100\rangle$, and for all simulated piston pressures along $\langle 110\rangle$. For instance, Fig. 3 shows one snapshot 4 ps after the shock for $P_{H} \sim 50 \mathrm{GPa}$. The kinetic energy map shows the layering of planes, alternating low and high kinetic energy regions, due to plane-plane collisions. A potential energy map shows the complimentary effect, as in a "harmonic oscillator", with lowest potential energy corresponding to highest kinetic energy and viceversa.

The varied behavior seen in Fig. 2 translates into widely different Hugoniot relationships along different orientations. Fig. 4 shows the MD results using the potential EAM2 for the shock Hugoniot along $\langle 100\rangle,\langle 110\rangle$ and $\langle 111\rangle$ directions. The experimental data for polycrystalline $\mathrm{Cu}$ for $U_{p}<4$ can be reasonably well fit by $U_{s}=3.96+1.5 U_{p}$ for the pressure range simulated [1-3], and this fit is also shown in Fig. 4. Below melting, the $\langle 100\rangle$ and $\langle 111\rangle$ curves have slopes much lower than those obtained from the LJ potential simulations. The slope of the Hugoniot does not change noticeably due to melting, which occurs in the range 200-220 $\mathrm{GPa}$ (2.0-2.2 Mbar), in agreement with experiments on polycrystalline $\mathrm{Cu}$ [43], with equilibrium "hydrostatic" MD simulations [24], and with a recent shock-release model of melting [44]. The higher values for the $\langle 110\rangle$ Hugoniot are related to the planeplane collisions that propagate the shock faster than along the other two directions. Using our simulation data and $\gamma_{\langle i j k\rangle}=2 s_{1\langle i j k\rangle}-1$, we have calculated the limiting value of the directional Grüneisen parameters. These values are 1.6, 3.4, and 3.1 for $\langle 100\rangle,\langle 110\rangle$ and $\langle 111\rangle$. For shock data of polycrystalline $\mathrm{Cu}$, the experimental isotropic Grüneisen parameter, 
$\overline{\gamma_{\langle i j k\rangle}}$, has been reported to be 1.99 [45], and 1.98 [15].

How can we relate our simulations to the many experimental results on polycrystalline Cu? There have been a number of studies using numerical continuum models of shock propagation through rectangular [46] or spherical grains [47], but unfortunately, different values of the shock velocities were not taken into account. A model numerical simulation of shock propagation through a polycrystalline slab with anisotropic shock velocities shows large deviations from the isotropic case, even for small anisotropies [48]. As an initial step, we have attempted to provide limiting bounds for polycrystalline $\mathrm{Cu}$ using the Voigt $\left({\overline{U_{s}}}_{\text {High }}\right)$ and Reuss $\left(\bar{U}_{s L o w}\right)$ averages of our single crystal results [21]. This analysis assumes that grain boundary effects can be neglected and that the grain distribution along directions other than $\langle 100\rangle,\langle 110\rangle$, and $\langle 111\rangle$ can be lumped into the distribution of these three directions. Fig. 5 shows the resulting shock velocity limits. In order to obtain the curves in Fig. 5, we fit the results from Fig. 4 to polynomials of $4^{\text {th }}$ order in the range $U_{p} \in(0.0,8.0)$ and use these polynomials to build the averages. A procedure similar to Voigt averaging has been used to estimate shock wave profiles in polycrystalline $\mathrm{Ni}$ [49]. Notice that the slope of the mean values is close to the best fit of experimental results, but appears shifted upwards for equal fractions of grains in the different directions. Increasing the $\langle 100\rangle$ fraction does lower the mean values, and this is consistent with $\langle 100\rangle$ being the preferred orientation in annealed polycrystalline $\mathrm{Cu}$, as confirmed by experiments [50]. Voigt and Reuss averaging differ by less than $2.5 \%$ for our simulations.

A calculation of the "isotropic" Hugoniot from Mitchell et al. [2] can be also seen in Fig. 5. Notice the change in slope of the Hugoniot at low velocities, which departs from both experiments and our simulation results. Since the energy behind the shock stays well below the Fermi energy for the piston velocities considered here, the electron thermal contribution to the EOS is small compared to the cold lattice pressure and the ion thermal (vibrational) pressure. Therefore, electron thermal conductivity will not affect the Hugoniot [51], and it is typically not included in a Grüneisen-type EOS. MD calculations including electronic thermal conduction and electron-phonon coupling through a two-temperature model (TTM) [52] are in progress, but for relatively small shock pressures the TTM contribution is small, as expected [53]. 


\section{SUMMARY}

We have presented MD simulations of the Hugoniot relationship for $\mathrm{Cu}$, for pressures in the range of 2 - $800 \mathrm{GPa}$ (20 kbar-8 MBar), which includes the melting transition. Our simulation results agree well with new experimental data for single crystal shocks along $\langle 100\rangle$ and $U_{p} \leq 1$. Qualitatively the results for pressures below melting agree with results from LJ solids [14], with several quantitative differences.

We observed that the shock velocity has a plateau, with $U_{s}=c_{o L}$, in the elastic region at low $U_{p}$. The HEL occurs at $32 \pm 2 \mathrm{GPa}$ for all studied directions, at a compression of $\sim 14 \%$ and a ratio $\left(U_{p} / c_{o L}\right) \sim 0.14-0.2$, similar to the LJ potential results [14]. Since simulations were carried out for perfect single crystals, the HEL from MD is much higher than the experimental value [39] and estimates based on a simple elasto-plastic model of the solid. Melting is found to happen in the range $200-220 \mathrm{GPa}$, in agreement with both experiments [43] and equilibirium MD simulations [24]. Melting happens at $\left(U_{s} / U_{p}\right) \sim 0.375$, i.e. $\left(U_{p} / c_{o L\langle i j k\rangle}\right)=0.5-0.75$, while for the LJ crystal $\left(U_{p} / c_{o L\langle i j k\rangle}\right) \sim 1$ at melting.

The slopes of the Hugoniot along $\langle 100\rangle$ and $\langle 111\rangle$ are much softer for EAM Cu than for the LJ system. The $\langle 110\rangle$ and $\langle 111\rangle$ results which were relatively close to each other for LJ in the strong shock regime below melting, now show large differences. As shock pressure increases, the Hugoniot curves for all directions do converge to the polycrystalline result, as expected, but the convergence happens near the melting pressure for the plastic front and at even higher pressures for the elastic precursor.

We also presented a simplified analysis of the average shock velocity for polycrystalline $\mathrm{Cu}$, and find that the value of the average Hugoniot is up to $20 \%$ larger than the experimental Hugoniot for polycrystals. This difference decreases if one assumes a dominant $\langle 100\rangle$ texture. This is consistent with the observation that a large contribution from $\langle 100\rangle$ texturing is required in polycrystalline experiments to account for the experimental value of the limiting Grüneisen parameter. The calculation of directional Grüneisen parameters for both pair and many-body potentials would allow a direct comparison of our limiting compression factors, and would pave the way for future anisotropic equations of state.

We have carried out some preliminary Hugoniot calculations for crystals including defects which decrease the HEL [40], and they seem to indicate that the elastic Hugoniot is not changed, while the plastic Hugoniot moves closer to the polycrystalline Hugoniot data. 
Even well annealed $\mathrm{Cu}$ single crystals have a dislocation density $10^{5}-10^{8} / \mathrm{cm}^{2}$, leading to a low experimental HEL [39]. Therefore, dislocation sources, and not texture, could be an alternative explanation to the departure of the calculated single crystal Hugoniot with respect to the polycrystal Hugoniot, even after averaging over different directions.

\section{Acknowledgments}

This work was performed under the auspices of the U. S. Department of Energy by the University of California, Lawrence Livermore National Laboratory under Contract No. W7405-Eng-48. We thank M. Kumar for access to his spall data, J. Moriarty for providing us the data from ref. [2], and J. Belak, J. Colvin, D. Kalantar, J. McNaney, R. Minich and B. Remington for useful discussions. 
[1] W. J. Nellis et al., Phys. Rev. Lett. 60, 1414 (1988).

[2] A.C. Mitchell et al., J. Appl. Phys. 69, 2981 (1991).

[3] W. J. Nellis et al., J. Appl. Phys. 93, 304 (2003).

[4] M.A. Meyers and L.E. Murr eds., Shock Waves and High Strain Rate Phenomena in Metals, Plenum, New York (1981).

[5] H.J. Frost and M.F. Ashby, Deformation Mechanism Maps, Pergamon Press, Oxford (1982).

[6] D.H. Kalantar et al., Phys. Plasmas 7, 1999 (2000).

[7] A. Loveridge-Smith et al., Phys. Rev. Lett. 86, 2349 (2000).

[8] M.A. Meyers et al., Acta Mater. 51, 1211 (2003).

[9] M. P. Allen and D. J. Tildesley, Computer Simulation of Liquids, Clarendon Press, New York, 1987.

[10] M. Shehadeh, H. Zbib, in preparation.

[11] B. L. Holian and P. S. Lomdahl, Science 80, 2085 (1998).

[12] V. V. Zhakhovskii, S. V. Zybin, K. Nishihara, and S. I. Anisimov, Phys. Rev. Lett. 83, 1175 (1999).

[13] J.-B. Maillet, M. Mareschal, L. Soulard, R. Ravelo, P. S. Lomdahl, T. C. Germann, and B. L. Holian, Phys. Rev. E 63, 016121 (2001).

[14] T. C. Germann, B. L. Holian, P. S. Lomdahl, and R. Ravelo, Phys. Rev. Lett. 84, 5351 (2000).

[15] Ya. B. Zeldovich and Yu. P. Raizer, Physics of Shock Waves and High Temperature Hydrodynamic Phenomena, W. D. Hayes and R. F. Probstein, eds., Dover, New York, 2002.

[16] D.S. Drumheller, Introduction to Wave Propagation in Nonlinear Fluids and Solids, Cambridge University Press, Cambridge, 1998.

[17] J.P. Hirth and J. Lothe, Theory of Dislocations, Wiley, New York, 1982.

[18] D. Wallace, Thermodynamics of crystals, John Wiley \& Sons, (1972).

[19] W. J. Drugan, J. Mech. Phys. Solids 46, 313 (1998).

[20] J. Lubliner, Plasticity Theory, Macmillan, New York (1990).

[21] G. Simmons, Single crystal elastic constants and calculated aggregate properties, a handbook, $2^{\text {nd }}$ ed., MTT press, Cambridge (1971).

[22] J. Diehl, Z. Metallk. 47, 331 (1956). 
[23] Y. Wang, D. Chen and X. Zhang, Phys. Rev. Lett. 84, 3220 (2000).

[24] J-W. Jeong and K.J. Chang, J. Phys. Condens. Matter 11, 3799 (1999).

[25] R.W. Minich, J.U. Cazamias, M. Kumar, and A.J. Schwartz, Met. Mater. Trans. A, submitted, 2003.

[26] T. Diaz de la Rubia and M.W. Guinan, Mat. Res. Forum 174, 151 (1990).

[27] E.M. Bringa et al., Nucl. Instr. Methods in Phys. Res. B 202(2003) 56.

[28] P.A. Taylor and B.W. Dodson, Phys. Rev. B 42, 1200 (1990).

[29] J. Belak, in Science and Technology of High Pressure, M.H. Manghnani, W.J. Nellis and M.F. Nicol eds., University Press, Hyderabad, 2000.

[30] A.I. Ryazanov, V.V. Dremov and M. Kiritani, Rad. Eff. and Def. in Solids 157, 209 (2002).

[31] O. Kum, J. Appl. Phys. 93, 3239(2003).

[32] A.J.E. Foreman, C.A. English and W.J. Phythian, Phil. Mag. 66, 655 (1992).

[33] Y. Mishin, M.J. Mehl, D.A. Papaconstantopoulos, A.F. Voter and J.D. Kress, Phys. Rev. B 63, 224106 (2001).

[34] C.B. Carter and I. L. F. Ray, Philos. Mag. 35, 189 (1977).

[35] L.E. Murr, Interfacial Phenomena in Metals and Alloys, Addison Wesley, Reading MA, 1975.

[36] E.M. Bringa, R. E. Johnson and M. Jakas, Phys. Rev. B 60, 15107 (1999).

[37] S.J. Zhou et al., Science 279, 1525 (1998).

[38] B.L. Holian et al., Phys. Rev. A 43, 2655 (1991).

[39] O.E. Jones and J. D. Mote, J. Appl. Phys. 40, 4920 (1969).

[40] L. Davila, P. Erhart, E. Bringa, M.A. Meyers, V.A. Lubarda, M.S. Schneider, R. Becker, and M. Kumar, submitted.

[41] O.E. Jones, in Metallurgical Effects and High Strain Rates, R.W. Rhode et al. eds., Plenum, New York (1973), pages 33-55.

[42] J.A. Zukas, T. Nicholas, H.F. Swift, and L.B. Greszczuk, Impact dynamics, Wiley, New York (1982).

[43] D. Hayes, R.S. Hixson and R.G. McQueen, CP505, Shock compression of condensed matter 1999, M.D. Furnish, L.C. Chhabildas, and R. S. Hixson, eds., American Institure of Physics, 2000, page 483 .

[44] C. Dai, H. Tan and H. Geng, J. Appl. Phys. 92, 5019 (2002).

[45] W.B. Holzapfel, Z. Kristallogr. 216, 473 (2001). 
[46] D.J. Benson, Wave Motion 21, 85 (1995)

[47] K. Kano and Y. Horie, Phys. Rev. B 59, 13672 (1999).

[48] R.C. Cook, Fusion Sci. Technol. 41, 155 (2002).

[49] M.A. Meyers, Mat. Sci. and Eng. 30, 99 (1977).

[50] F.J. Humphreys and M. Hatherly, Recrystallization and related annealing phenomena, Pergamon, Oxford (1995).

[51] J. Colvin it. et al., in Proc. of the 6th International Workshop on the Physics of Compressible Turbulent Mixing, G. Jourdan \& L. Houas, eds., Universite de Provence, Marseille, 1997.

[52] D. Ivanov and L. Zhigilei, Phys. Rev. Lett. 91, 105701 (2003).

[53] B. Remington et al., Met. Mat. Trans. A, in press. 
TABLE I: Parameters for the potentials discussed in the text. " $r_{c u t}$ shell" indicates the last shell of neigbors included by the potential cut-off. $d_{S F}$ is the equilibrium separation between $\mathrm{SF}$ at $\mathrm{P}=0$.

\begin{tabular}{lllll}
\hline \hline Potential & LJ $^{a}$ & EAM1 & EAM2 & Exp. \\
\hline$r_{\text {cut }}$ shell & $2^{n d}$ & $3^{\text {rd }}$ & $4^{\text {th }}$ & - \\
$\gamma_{S F}\left(\mathrm{~mJ} / \mathrm{m}^{2}\right)$ & 0 & 11.4 & 44.0 & $45.0^{b}-78.0^{c}$ \\
$d_{S F}(\mathrm{~nm})$ & $\infty$ & $\sim 8$ & $\sim 2$ & $\sim 2-1$ \\
\hline \hline
\end{tabular}

${ }^{a}$ Ref. 14.

${ }^{b}$ Ref. 34 .

${ }^{c}$ Ref. 35 . 
FIG. 1: Shock velocity versus piston velocity along $\langle 100\rangle$. MD simulations for different potentials: EAM1 (solid squares), EAM2 (open squares), and Morse potential (diamonds). Two single points for underdriven plastic shock waves were omitted for clarity for the EAM2 potential. Experimental points (up triangles) are also shown, together with linear fits to LJ potential simulations [14] (solid line), and the EAM2 simulations [fit in the interval $\left.U_{p}=(0,1.5)\right]$ (dashed line).

FIG. 2: Snapshots of velocity profiles for shock waves along $\langle 100\rangle,\langle 110\rangle$, and $\langle 111\rangle$ (from top to bottom), taken 3 ps after the shock was started, for a piston pressure of 100 GPa. Dashed lines give approximate location of plastic front.

FIG. 3: Snapshots of a shock along $\langle 110\rangle$, showing the elastic precursors and the plastic wave. Coloring proportional to the kinetic energy of the atoms. The volume of the simulated sample was roughly $25 a_{o} \times 25 a_{o} \times 100 a_{o}$, with $a_{o}=3.615 \AA$. 
FIG. 4: Shock velocity versus piston velocity along different crystalline directions: $\langle 100\rangle,\langle 110\rangle$, and $\langle 111\rangle$. Both elastic and plastic velocities are shown. An isobar at $220 \mathrm{GPa}$ gives a rough estimate of melting (dashed line). Large error bars for the elastic shock above melting for $\langle 110\rangle$ are due to a short-lived elastic precursor that is promptly overrun by the melting wave. Dislocation production, and therefore a plastic shock wave, was observed for $U_{p}=0.75 \mathrm{~km} / \mathrm{s}$ along the $\langle 111\rangle$ direction (arrow shown), but an accurate value for the plastic shock velocity for that $U_{p}$ was not obtained.

FIG. 5: Shock velocity versus piston velocity using Voigt and Reuss averaging over different crystalline directions, for two different textures. Fit to experimental data for polycrystals, $U_{s}=3.96+1.5 U_{p}$ (solid line), and "isotropic" EOS calculations [2] (squares) are also shown. 


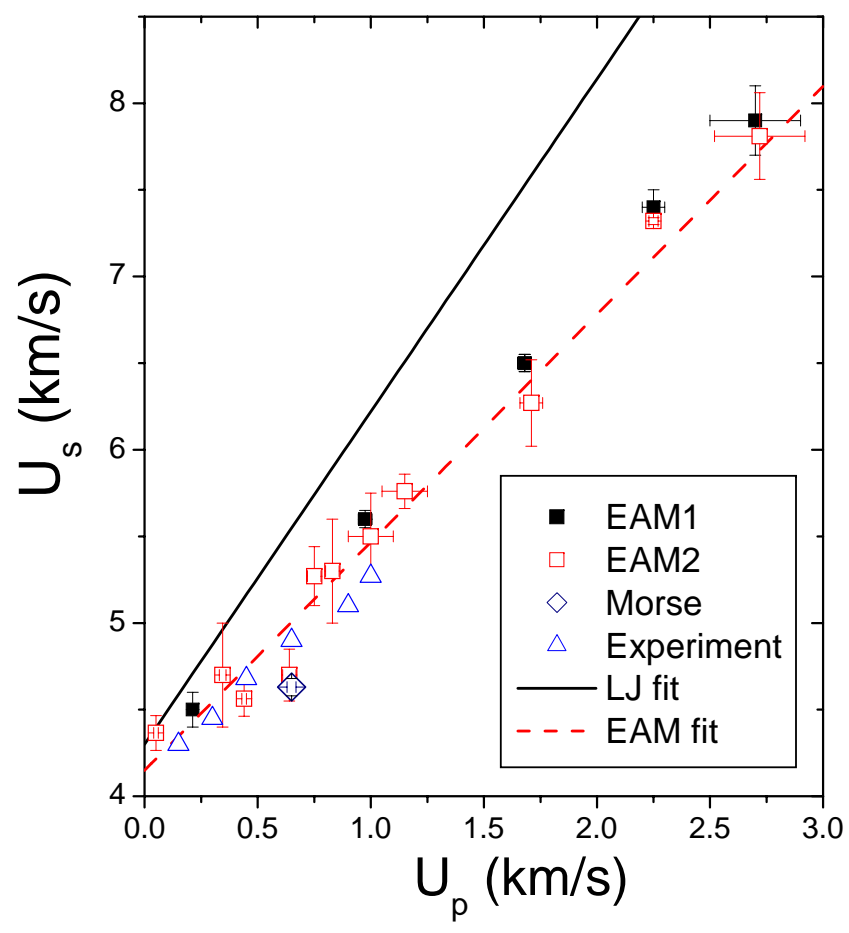

Bringa et al., Fig. 1 


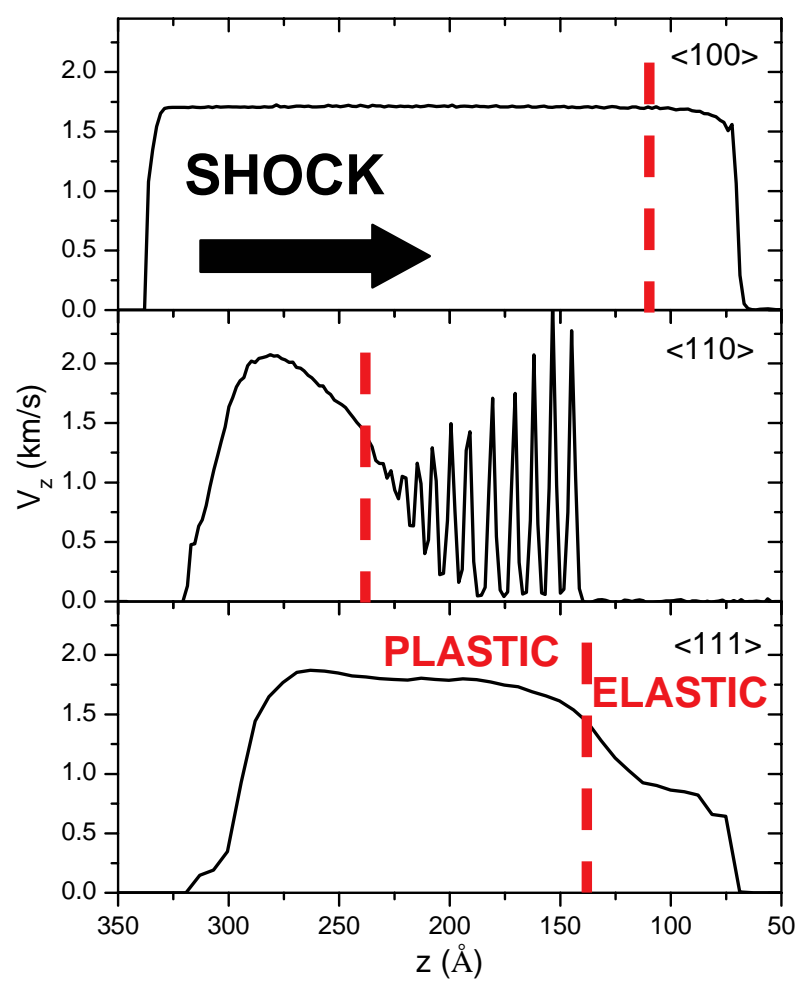

Bringa et al., Fig. 2 


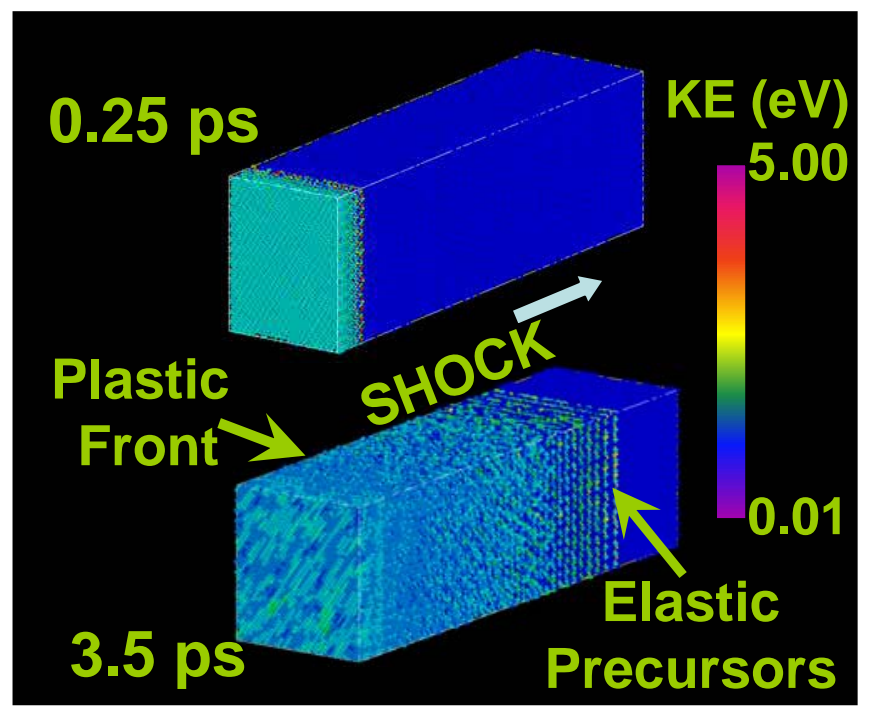

Bringa et al., Fig. 3 


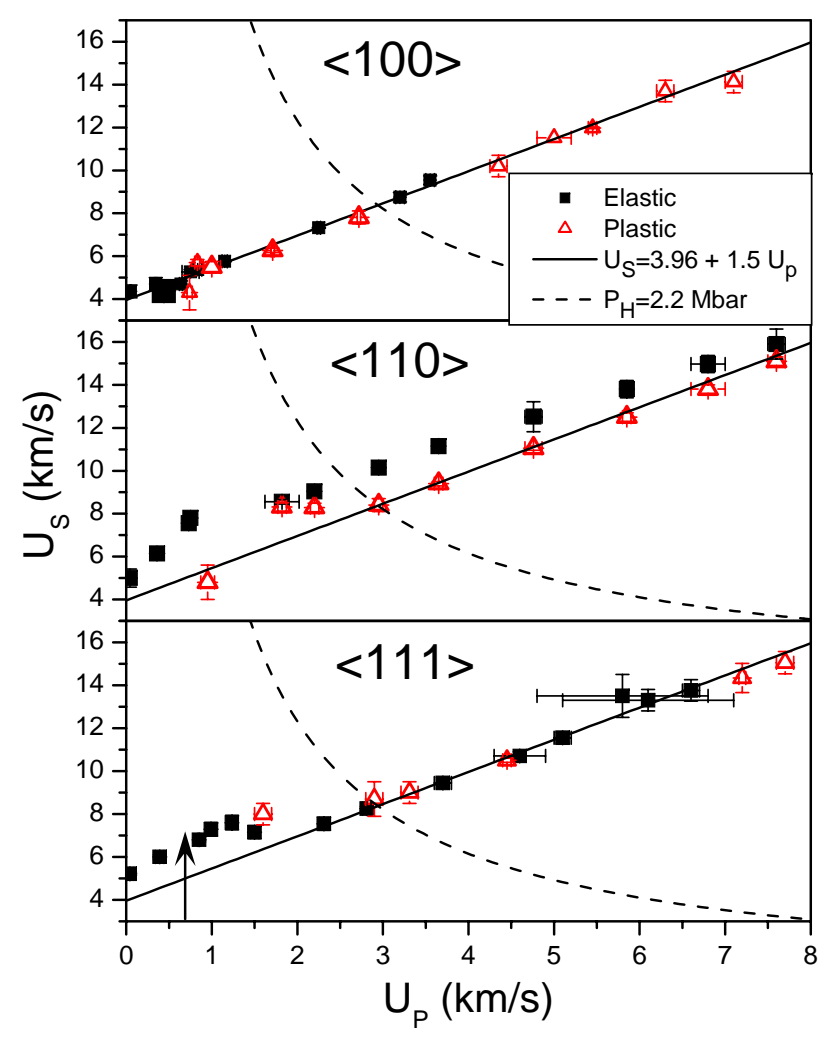

\section{Bringa et al., Fig. 4}




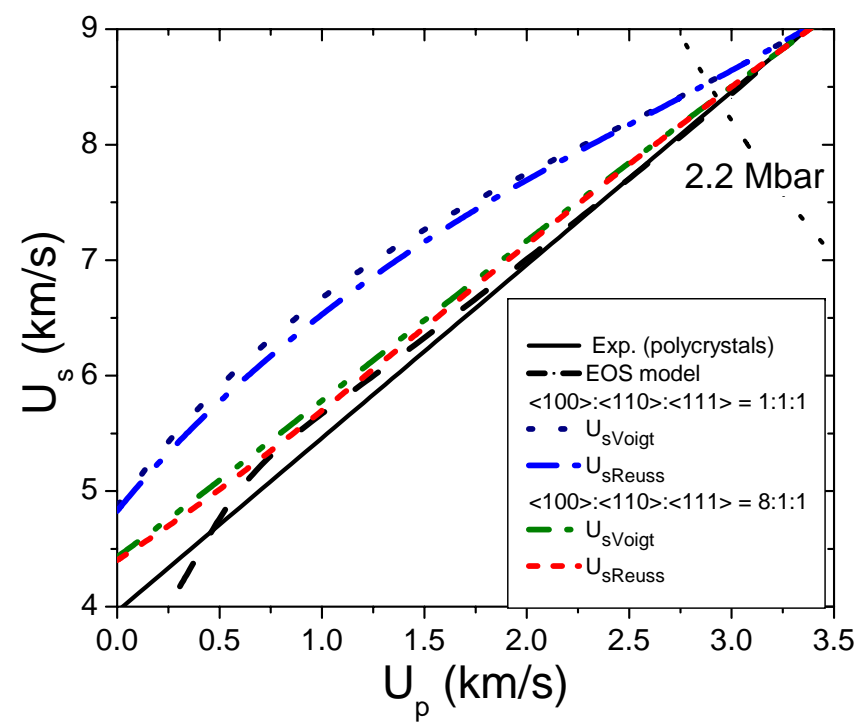

Bringa et al., Fig. 5 